\title{
El reto de la Evaluación del Impacto Social de la Tecnología en España
}

\author{
Francisco Javier GÓMEZ GONZÁLEZ \\ Universidad de Valladolid \\ javier@emp.uva.es
}

\author{
Cristina DURLAN \\ Universidad de Valladolid \\ cdurlan@emp.uva.es
}

Recibido: 31-05-2013

Aceptado: 13-05-2014

\author{
Guillermo ALEIXANDRE MENDIZÁBAL \\ Universidad de Valladolid \\ galeixam@eco.uva.es \\ galeixam@eco.uva.es
}

\begin{abstract}
Resumen:
La fuerte interacción existente entre los procesos tecnológicos y la sociedad requiere la puesta en marcha de dinámicas que permitan la evaluación del impacto social de la tecnología que se crea y/o se utiliza en un determinado país. Fruto de esta necesidad, ha visto la luz un significativo volumen de experiencias y trabajos relacionados con el impacto social realizados en el contexto internacional, constatándose también la existencia de una incipiente dinámica en esta materia en el marco español. El presente trabajo asume el objetivo de analizar el grado de incorporación de la evaluación del impacto social de la tecnología en España y, a partir de este análisis, plantear pautas de actuación futuras encaminadas a su promoción.

Para alcanzar este objetivo, en primer lugar, se presentan los diferentes conceptos que permiten acotar el ámbito de la evaluación del impacto social de las actividades y proyectos de carácter tecnológico. En segundo lugar, y tras valorar de manera global el nivel de desarrollo de este tipo de evaluación en nuestro país, se describen diversas líneas de trabajo directamente relacionadas con ella y que se están desarrollando actualmente en España. Partiendo de este marco, se comparan estas aportaciones para mostrar la situación del impacto social de la tecnología en España, constatándose la existencia de contribuciones heterogéneas, con un enfoque diverso, distintos grados de adopción y consolidación y con una dispar implicación del sector público; presentando, en su conjunto, un nivel de desarrollo relativamente débil.
\end{abstract}

Palabras clave: evaluación del impacto social, tecnología, evaluación de tecnologías, responsabilidad social corporativa, evaluación del impacto ambiental 


\title{
The Challenge of Social Impact Assessment of Technology in Spain
}

\begin{abstract}
The strong interaction between technological processes and society requires the implementation of a series of practices that allow the assessment of the social impact of the technology created and/or used in a country. As a result of this need, a significant amount of experiences and studies has appeared in the international context and, in a less extend, in Spain.

The aim of this paper is to analyse the incorporation of social impact assessment of technology in the Spanish context and, hence, to identify future trends of action in this area.

Therefore, firstly, we introduce several concepts that allow limiting the scope of the social impact assessment of the technological activities and projects. Secondly, after a brief evaluation of the deployment of social impact assessment of technological activities and projects in our country, we extend the scope of analysis in order to describe various lines of work developed in Spain that are related to this type of assessment. Based on the previous review, we compare these contributions to conclude that the Spanish studies on the social impact assessment of technology are characterized by the existence of some heterogeneous inputs with different approaches, different levels of adoption and consolidation and different degrees of public sector involvement. In general, the level of development of the evaluation of the social impact assessment of technological activities and projects is relatively weak.
\end{abstract}

Keywords: social impact assessment, technology assessment, corporate social responsibility, environmental assessments

\section{Referencia normalizada}

Gómez González, F.J. et.al.. (2014). "El reto de la Evaluación del Impacto Social de la Tecnología en España”. Política y Sociedad, Vol 51, Núm. 2: 447-480

Sumario: Introducción. 1.Delimitación y justificación del objeto de estudio. 2.La evaluación del impacto social de la tecnología en España. 3.Reflexiones sobre la evaluación del impacto social de actividades tecnológicas en España. 4.Conclusiones y consideraciones para el futuro. 


\section{Introducción}

En los países del contexto europeo y norteamericano se han desarrollado en las últimas décadas dinámicas encaminadas a fomentar y difundir la evaluación del impacto social de las actuaciones humanas (Becker y Vanclay, 2003; Esteves et al., 2012). Un número importante de esas actuaciones tienen un significativo contenido tecnológico que también requeriría una evaluación de su impacto social (Labuschagne y Brent, 2006; Schmidt et al., 2004; Manhart y Griesshammer, 2006).

La razón de partida para argumentar la necesidad y pertinencia de estas iniciativas es la fuerte interacción que existe entre los procesos tecnológicos y la sociedad en la que se llevan a cabo estos procesos. En ese contexto, es importante subrayar que la evaluación del impacto social de la tecnología favorece la rendición de cuentas de entidades públicas y privadas, facilita la toma de decisiones y la distribución de recursos así como la evaluación estratégica derivada del aprendizaje tanto a nivel político como de gestión (Rip, 2003). Como consecuencia de todas estas aportaciones, la evaluación del impacto social de la tecnología constituye actualmente una práctica con una presencia creciente a nivel internacional, lo que hace pertinente un análisis del nivel de utilización de esta metodología en el contexto español.

Aunque estas dinámicas de evaluación se han aplicado con diferentes perspectivas y niveles de globalidad, incluyendo el diagnóstico y valoración de alternativas y políticas tecnológicas, parece innegable la necesidad de dar protagonismo al enfoque de actividades y proyectos, puesto que una evaluación del impacto centrada en este nivel incrementa la información disponible por parte de los tecnólogos y los agentes implicados en el desarrollo concreto de la tecnología, y de esta manera, apoya la construcción de una tecnología social y responsable.

El Comité Consultivo Europeo de Investigación (EURAB, 2005) señala la importancia de la intervención en la evaluación y gestión de proyectos para mejorar la tecnología e incrementar su incidencia en el bienestar y el desarrollo. De manera similar, resalta la necesidad de mejorar el gobierno y gestión de los proyectos como estrategia para una mejor gobernanza de la ciencia y la tecnología.

En coherencia con la tendencia señalada, el presente trabajo asume el objetivo de constatar el grado de avance que ha experimentado la evaluación del impacto social de actividades y proyectos tecnológicos en España y, a partir de este análisis, plantear pautas de actuación futuras en dicho ámbito.

Con ese objetivo, el artículo se estructura en cuatro apartados: en primer lugar, se presentan aspectos relacionados con el concepto y el proceso de evaluación del impacto social de las actividades y proyectos de carácter tecnológico; en segundo lugar, y tras valorar de manera global el nivel de desarrollo de este tipo de evaluación en nuestro país, se describen diversas líneas de trabajo llevadas a cabo en España que están relacionadas de alguna forma con ella; en tercer lugar, se analizan las aportaciones de estas líneas en la consolidación de la evaluación del impacto social de actividades y proyectos tecnológicos; y finalmente, se presentan las con- 
clusiones de este trabajo y algunas consideraciones sobre el desarrollo futuro de las dinámicas en este campo.

\section{Delimitación y justificación del objeto de estudio}

Como paso preliminar al análisis del grado de incorporación de las dinámicas de evaluación del impacto social de las actividades tecnológicas en España, es conveniente establecer algunas precisiones relativas al objeto de análisis. En este sentido, a continuación se detalla el significado de los tres términos empleados en este trabajo: impacto social, tecnología y evaluación del impacto social.

Para definir el término impacto social se parte de las aportaciones de la Asociación Internacional para la Evaluación del Impacto (IAIA), que entienden por esta expresión:

"las consecuencias para la población humana de cualquier acción pública o privada que altera la forma en que las personas viven, trabajan, se entretienen, se relacionan con los otros, se organizan para satisfacer sus necesidades y se las arreglan como miembros de la sociedad. El término también incluye el impacto cultural que involucra cambios en las normas, valores y creencias que guían y racionalizan el conocimiento de ellos mismos y de la sociedad" (Interorganizational Committee on Principles and Guidelines for Social Impact Assessment, 2003, p. 231).

Conviene precisar que, a pesar de su uso generalizado, el término impacto social presenta importantes debates y controversias, como han reflejado, para el caso latinoamericano Albornoz et al. (2005) y Estébanez (2003). Por lo que se refiere al concepto de impacto, éste es relativamente ambiguo desde el punto de vista semántico, puesto que en algunos casos el uso del lenguaje le otorga un matiz de efecto negativo y en otros neutro. En muchos casos se confunde impacto con resultado directo, mientras que en otros se considera como un efecto indirecto, consecuencia no prevista, diferida, etc.

Además, la idea de impacto también presenta un matiz semántico de carácter mecanicista y unidireccional que puede condicionar su interpretación. Así, Luján y López Cerezo (2003) al definir las fases que describen la evolución del pensamiento reciente sobre las consecuencias sociales de la tecnología, señalan que la primera fase estuvo protagonizada por la idea de impacto social, concebida como un efecto negativo y partiendo de una cierta posición de autonomía de la tecnología respecto a la sociedad, la segunda por la idea de riesgo y la tercera por la idea de incertidumbre. Esta evolución expresa que el concepto de impacto, tiene una importante carga valorativa que establece matices diferenciales con otros conceptos como riesgo o invasión (Lizcano, 1996).

Por lo que se refiere al término social utilizado dentro de la definición de impacto social, debe señalarse que la Sociología ha experimentado tradicionalmente importantes dificultades para definir adecuadamente la dimensión social frente al resto de dimensiones que articulan el desarrollo de las sociedades. Una parte importante de estas dificultades se deriva del carácter polisémico del término social, que 
se usa tanto para hacer referencia al marco general de las sociedades como a un aspecto concreto dentro de ellas: la llamada dimensión social o societal, basada en las estructuras de relación e interacción entre grupos y que presenta diferencias con los aspectos económicos, culturales, laborales, ambientales, etc.

En este trabajo, sin poner en cuestión estas interpretaciones, se asume el término impacto social en la acepción definida por la IAIA, anteriormente citada, y que en la actualidad domina una parte importante de la literatura relacionada con la evaluación del impacto.

A su vez, el impacto social en este trabajo se circunscribe al ámbito de las actividades tecnológicas, por lo que es conveniente definir el concepto de tecnología. Con un carácter globalizador la tecnología puede ser entendida como:

"un conjunto de conocimientos de base científica que permiten describir, explicar, diseñar y aplicar soluciones técnicas a problemas prácticos de forma sistemática y racional” (Quintanilla, 1998).

Así, un proyecto o actividad tecnológica sería un sistema de acciones socialmente estructuradas, acotadas en el tiempo, orientadas a los procesos productivos industriales y estrechamente vinculadas al conocimiento científico.

Por último, para caracterizar la evaluación del impacto social de una actividad tecnológica puede acudirse a la definición de evaluación del impacto social siguiendo, en este caso, la propuesta de Vanclay (2003), que posteriormente fue aceptada por la IAIA, y que la interpreta como un tipo de actividad que:

"incluye los procesos de análisis, monitorización y gestión de las consecuencias sociales intencionadas o no intencionadas, tanto positivas como negativas, de intervenciones planificadas (políticas, programas, planes, proyectos) y cualquier proceso de cambio social invocado por dichas intervenciones. Su propósito principal es conseguir un entorno humano y biofísico más sostenible e igualitario” (Vanclay, 2003, p. $6)$.

En el presente artículo se asume que la evaluación del impacto social de una actividad tecnológica comprende aquellas metodologías que permitan analizar y valorar las repercusiones que las actividades y los proyectos que generan y/o usan tecnología tienen sobre la sociedad en su conjunto, aunque siempre valorando de manera más significativa los aspectos culturales y sociales, debido a que la evaluación del impacto económico ya cuenta con una trayectoria muy consolidada y un cuerpo de metodología propia.

\section{La Evaluación del impacto Social de la tecnología en España}

A la hora de analizar la incorporación de la evaluación del impacto social de la tecnología en el conjunto de prácticas evaluativas en España, hay que tener en consideración las singularidades del sistema tecnológico español. De forma muy sintética y tal y como quedan recogidas en diversos informes (OCDE, 2010; Fundación COTEC, 2011; FECYT, 2006), estas singularidades se expresan en el hecho de 
que España cuenta con un nivel intermedio de gasto en $\mathrm{I}+\mathrm{D}$, con una mayor presencia del sector público y un elevado nivel de excelencia científica, pero con una escasa generación de resultados tecnológicos. Además de estas singularidades, España se caracteriza por una débil posición como productor de tecnología, siendo más importante su papel como consumidor, como muestra el hecho de que tanto la balanza de pagos tecnológica como la balanza de pagos de productos tecnológicos mantienen de manera continuada una situación deficitaria (Banco de España, 2012). Esta realidad puede tener asociada la aceptación de la tecnología como algo dado, externo, sobre la cual no se tiene capacidad para actuar desde la posición del ciudadano, puesto que su producción es externa y lejana. Aunque es muy difícil atribuir causas a este fenómeno, es importante señalar que España cuenta con uno de los mayores niveles de optimismo y confianza en la ciencia y la tecnología dentro de los países europeos (Eurostat, 2010).

Por otra parte, España no cuenta con una práctica consolidada de rendición de cuentas a los ciudadanos sobre la acción pública (Jiménez Hernández, 2009) o empresarial (Cantó-Milà y Lozano, 2009).

De manera coherente con las consideraciones anteriores, puede constatarse que la evaluación del impacto social de actividades y proyectos tecnológicos en España no constituye una línea de investigación claramente identificable dentro de la comunidad científica, aunque existen aportaciones puntuales en este campo (Moñuz Chércoles et al., 2003, López Cerezo y Luján, 2002, Osuna et al., 2003).

Estas aportaciones reflejan que este tipo de evaluación puede llevarse a cabo en diferentes etapas del proceso de realización del proyecto, distinguiéndose: la fase ex-ante, el diseño y aprobación; la fase in-itinere en la ejecución y supervisión; y la fase ex-post, en la etapa de evaluación. Dentro de estas alternativas, en el ámbito estatal y para los proyectos de I+D e innovación, predomina la evaluación ex-ante por pares asociada a los procedimientos de financiación y selección de proyectos que valoran la calidad, la oportunidad y las posibilidades de rendimiento científico futuro de los proyectos (Fernández Esquinas et al., 2011).

En la práctica evaluativa pública de proyectos, los principales actores son la Agencia Nacional de Evaluación y Prospectiva (ANEP) y el Centro para el Desarrollo Tecnológico Industrial (CDTI), y su actuación se orienta no tanto hacia el impacto social de la ciencia y la tecnológica como hacia la excelencia científica, en el primer caso, y la promoción de la innovación y el desarrollo tecnológico, en el segundo.

Frente a esta cierta debilidad de las prácticas de evaluación del impacto social de los proyectos tecnológicos, existe un cierto volumen de trabajo en campos afines y/o trasversales en los cuales está presente un enfoque similar al presentado por la IAIA y que plantean iniciativas de interés para el desarrollo de procesos de evaluación del impacto social de intervenciones tecnológicas. Esta afinidad justifica su análisis dentro de un trabajo destinado a comprender el estado de la evaluación del impacto social de proyectos tecnológicos.

Con la intención de explorar esas aportaciones, el presente trabajo amplía el ámbito de análisis de la evaluación en dos dimensiones: el objeto de la evaluación y la 
orientación de la evaluación. En el primer caso se amplía desde las evaluaciones que se centran en proyectos concretos, con un enfoque micro, hasta evaluaciones con un objeto más amplio, como puede ser organizaciones, políticas o tecnologías, con una aproximación meso y macro.

En cuanto a la segunda dimensión se amplía desde un planteamiento que considera únicamente la evaluación de proyectos tecnológicos hasta aquellos planteamientos que tienen en consideración otras evaluaciones con una orientación más general, donde los aspectos tecnológicos serán un elemento más en un conjunto más amplio, como puede ser el caso de la evaluación del impacto ambiental o de proyectos de cooperación. En ellos, la presencia de contenido tecnológico es muy dispar, desde su inexistencia, como en un programa de ayuda con alimentos, hasta llegar a ser un elemento esencial, como en el caso de un proyecto de cooperación para la electrificación de una comunidad empleando energías renovables.

Considerando simultáneamente las dos dimensiones citadas pueden plantearse tres ampliaciones del análisis (Tabla 1), contando cada una de ellas con tradiciones metodológicas que cuentan con aportaciones interesantes para la evaluación del impacto social de proyectos tecnológicos. A continuación se enumeran estas tradiciones:

1. Tradiciones o enfoques metodológicos que cuentan con una orientación tecnológica y un objeto de análisis amplio, tales como planes o políticas, que asumen como objetivo analizar el impacto de la tecnología desde distintas perspectivas, como son la económica, la medioambiental o la social. Dentro de este cuadrante se encontrarían: la evaluación de tecnologías, la evaluación de políticas de I+D+i o los estudios de ciencia, tecnología y sociedad. Como ejemplo puede citarse el análisis de las consecuencias de los servicios en internet (STOA, 2014).

2. Tradiciones o enfoques metodológicos que cuentan con una orientación general y un objeto de evaluación definido a nivel de proyecto. Este es el caso, por ejemplo, de la evaluación del impacto ambiental o la evaluación de proyectos de cooperación. Aunque la orientación es de carácter general, en algunos de estos campos la componente tecnológica del proyecto puede tener cierta importancia, este sería el caso, por ejemplo, de un proyecto de cooperación al desarrollo vinculado con energías renovables (Ingeniería sin Fronteras, 1999).

3. Tradiciones o enfoques metodológicos que cuentan con una orientación general y un enfoque de carácter amplio, como ocurre en el caso de las evaluaciones vinculadas con la realización de memorias de responsabilidad social corporativa, que parte de modelos de evaluación centrados en la actividad y resultados de organizaciones y pueden ser un punto de partida para la inclusión de aspectos relativos al impacto social de las tecnologías que maneja o produce dicha organización. Por ejemplo, la elaboración de informes sobre responsabilidad social corporativa de empresas de fabricación de equipos electrónicos (NOKIA, 2012). 
Tabla 1. Ámbitos de trabajo de interés para la evaluación del impacto social de la tecnología por objeto y orientación

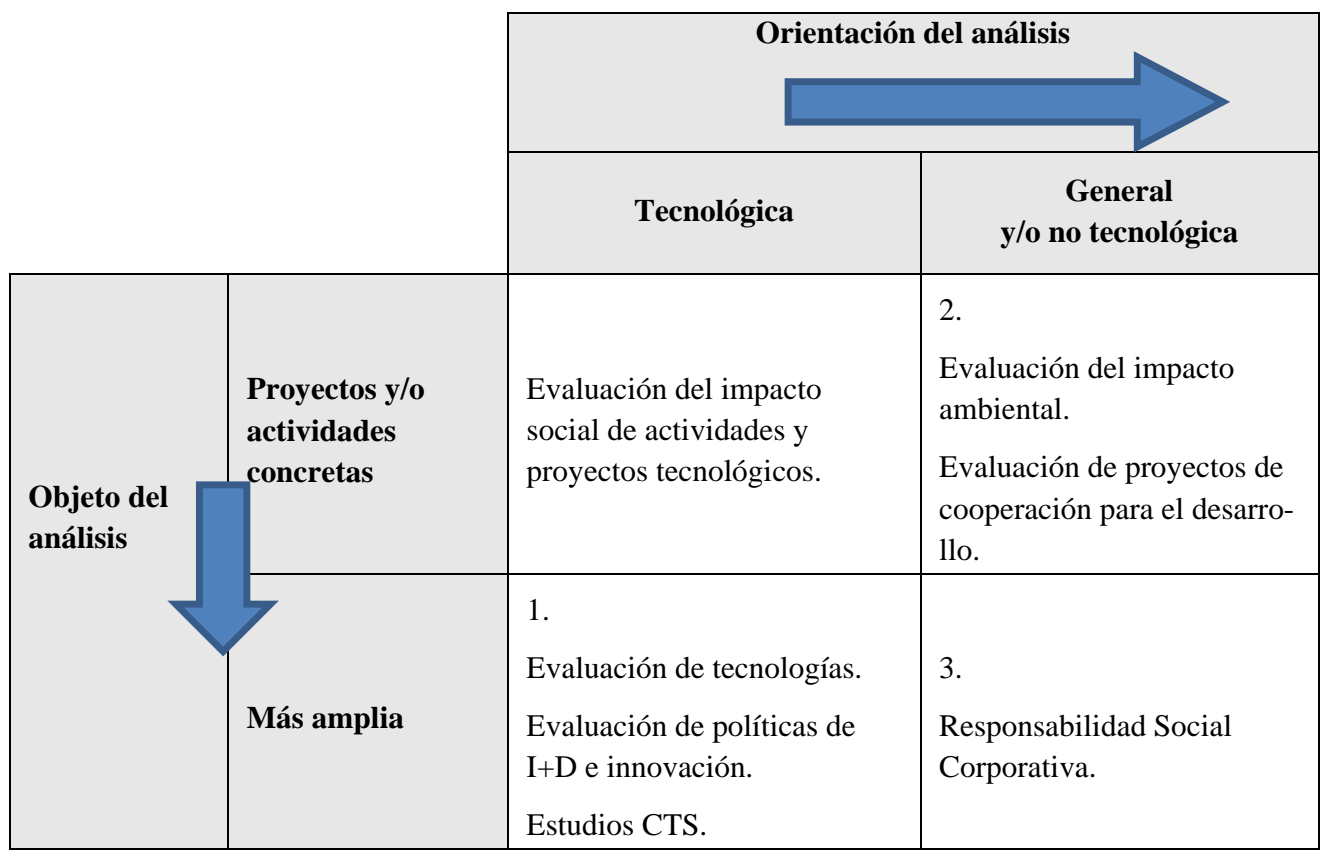

Fuente: elaboración propia.

Tal como recoge la Tabla 1, dentro de estas tres extensiones se han identificado seis ámbitos de trabajo que actúan como potenciadores de las prácticas de evaluación del impacto social de la tecnología en España y que se describen en los próximos epígrafes. Se trata de perspectivas complementarias y heterogéneas, entre las cuales están presentes experiencias pioneras, campos estratégicos de aplicación y, también, campos con cierta sensibilidad hacia el tema del impacto social de la tecnología pero que no han desarrollado un cuerpo formal teórico o una metodología específica para su evaluación.

\subsection{La Evaluación de Tecnologías}

La Evaluación de Tecnologías (Technology Assessment) hace referencia a un marco evaluativo que supera en perspectiva a la evaluación del impacto social de las actividades tecnológicas, y que asume un perspectiva más global. Esta evaluación surge en la década de 1960 fruto de la toma de conciencia, por parte de políticos y gestores vinculados al Congreso de Estados Unidos, de los profundos efectos asociados a las decisiones tecnológicas, planteándose la necesidad de establecer algún mecanismo de alerta temprana sobre las consecuencias de las invenciones, que 
tuviera un funcionamiento imparcial y permitiera mejorar la toma de decisiones del Congreso en el ámbito de los programas tecnológicos (Chedd, 1973).

Esta formulación del análisis de las tecnologías se institucionaliza en los Estados Unidos con la creación de la Office of Technology Assessment (OTA) en 1978 (Porter, 1995) ${ }^{1}$. Europa siguió la senda marcada al otro lado del Atlántico a través de la creación de distintas iniciativas a escala nacional y europea ${ }^{2}$.

Este tipo de evaluación puede entenderse como un proceso científico, interactivo y comunicativo que tiene como objetivo contribuir a la formación de la opinión pública y política sobre los aspectos sociales de la ciencia y la tecnología ${ }^{3}$. A partir de su planteamiento inicial, se ha producido una importante transformación de esta evaluación en función de las especificidades de su desarrollo formulándose, entre otras, las siguientes formas de Evaluación de Tecnologías: parlamentaria, experta, participativa, constructiva o discursiva (Decker y Ladikas, 2004).

Mientras que en el conjunto de Europa existe una actividad identificable en relación con la Evaluación de Tecnologías, en España su desarrollo ha sido claramente menor, con experiencias de interés pero poco generalizadas. Entre ellas, puede destacarse como iniciativa pionera la creación en 1998 del Consejo Asesor del Parlamento de Cataluña en materia Científica y Tecnológica (CAPCIT), que ha trabajado sobre temas como cambio climático y economía, transgénicos o el virus del papiloma humano. Llama la atención este reducido desarrollo si se considera que el sistema público español ha contado con actores privilegiados que podrían haber asumido esta tarea de evaluación, entre los cuales pueden señalarse a los siguientes (Muñoz-Alonso, 1997):

- La ANEP, previamente citada, que asume entre otras funciones, la realización de estudios prospectivos en materia de investigación científica y desarrollo tecnológico.

- Los Consejos Asesores, que comenzaron su andadura con el Consejo Asesor para la Ciencia y la Tecnología, desarrollado a partir de la Ley de Ciencia de 1986 con la función de gestionar la participación de la comunidad científica y de los agentes económicos y sociales en la elaboración, seguimiento y evalua-

${ }^{1}$ La Office of Technology Assessment estuvo operativa entre 1972 y 1995, siendo su finalidad proporcionar a los miembros del Congreso americano y de sus Comités un análisis objetivo sobre cuestiones científicas y técnicas complejas.

${ }^{2}$ Entre los organismos con una mayor trayectoria en evaluación de tecnologías en Europa pueden citarse: la Danish Board of Technology (DBT) danesa, el Flemish Institute for Science and Technology Assessment (viWTA) belga, la Office of Technology Assessment (TAB) alemana o, para el conjunto europeo, la Science and Technology Options Assessment (STOA), el European Technology Assessment Group (ETAG) o la European Parliamentary Technology Assessment (EPTA).

${ }^{3}$ Definición propuesta en el proyecto TAMI (Technology assessment in Europe; between method and impact) desarrollado entre 2002 y 2003 y financiado por el V Programa Marco de I+D de la Unión Europea. 
ción del Plan Nacional de I+D. Con posterioridad, la ley 14/2011, de 1 de junio, de la ciencia, la tecnología y la innovación mantiene un marco institucional que posibilita la evaluación de tecnologías, principalmente a través de la función asignada al Consejo de Política Científica y Tecnológica de "emitir los informes y dictámenes que le sean solicitados por el Gobierno o por las Comunidades Autónomas" (Art.8) o la función encomendada al Consejo Asesor de Ciencia, Tecnología e Innovación de "promover la introducción en el Sistema Español de Ciencia, Tecnología e innovación de mecanismos rigurosos de evaluación que permitan medir la eficacia social de los recursos públicos utilizados” (Art.9). Además, el Comité Español de Ética de la Investigación puede "emitir informes, propuestas y recomendaciones sobre materias relacionadas con la ética profesional en la investigación científica y técnica” (Art. 10).

- La Comisión Permanente Legislativa dedicada a temas de investigación científica y desarrollo tecnológico en el Parlamento.

También es posible identificar otros actores que realizan Evaluaciones de Tecnologías de corte sectorial, como es el caso de Fundesco en el ámbito de la sociedad de la información, el OPTI en el sector industrial, o distintas agencias en el área de la salud.

Los trabajos realizados en el ámbito de la Evaluación de Tecnologías ofrecen un marco general de carácter estratégico para la evaluación del impacto social de un proyecto tecnológico particular. Además, aportan una amplia gama de metodologías y herramientas de evaluación (Tran, 2007; Decker y Ladikas, 2004) y son una fuente de información valiosa a la hora de abordar la evaluación de proyectos porque permite determinar tanto impactos como posibles indicadores asociados.

\subsection{El análisis de la evaluación de políticas de $I+D$ e innovación}

Este epígrafe se centra en una tradición evaluativa específica: la evaluación de políticas públicas, en la cual la dimensión social de las actuaciones queda explícitamente recogida en documentos de referencia nacionales y europeos.

Así, la Estrategia Española de Ciencia y Tecnología y de Innovación 2013-2020 reconoce que la acción pública debe estar orientada a la obtención de resultados que aceleren el impacto social y económico de las actividades de I+D e innovación y la necesidad de aplicar criterios de impacto social en la asignación de los recursos públicos. Este planteamiento sigue la senda establecida en la Estrategia precedente y está en línea con las declaraciones políticas en el ámbito de la Unión Europea ${ }^{4}$.

\footnotetext{
${ }^{4}$ De forma similar, en el ámbito comunitario, la Comisión Europea en la comunicación “Europa 2020” pone de manifiesto el papel de las actividades de I+D de innovación en la construcción europea, persiguiendo "una economía con un alto nivel de empleo que redunde
} 
Como consecuencia de lo anterior, sería razonable la existencia de estudios y metodologías orientadas a conocer la manera en que la acción de los gestores públicos ha afectado a la vida de sus ciudadanos.

Para analizar la evaluación del impacto social de las políticas de I+D es necesario hacer referencia al proceso de diseño, ejecución y evaluación de estas políticas. Tras la entrada en vigor de la Ley de la Ciencia, la Tecnología y la Innovación de 2011 y los ajustes ministeriales posteriores ${ }^{5}$, las líneas maestras de política científica y tecnológica española se plasman en la Estrategia Española de Ciencia y Tecnología y de Innovación que elabora la Secretaría de Estado de Investigación, Desarrollo e Innovación, adscrita al Ministerio de Economía y Competitividad, en colaboración con el Consejo de Política Científica, Tecnológica y de Innovación, y posteriormente se traslada a un Plan Estatal de Investigación Científica y Técnica y de Innovación, con un horizonte temporal de medio plazo. Éste, a su vez, da lugar a un Plan de Actuación Anual con su reflejo en partidas concretas en los Presupuestos Generales de Estado. Además, se elaboran Memorias de Actividades de $I+D+i$ anuales que ofrecen una visión conjunta de las actividades financiadas públicamente en este ámbito. El Sistema integral de seguimiento y evaluación analiza y evalúa los instrumentos y actuaciones del Plan de Actuación para proponer recomendaciones. En general, la evaluación se centra en el análisis de indicadores de recursos y de resultados científico-tecnológicos, sin abordar el impacto social de las actuaciones desarrolladas. Además de esta evaluación técnica, existe un control político a través de las comparecencias en el Parlamento y un control administrativo a través del Tribunal de Cuentas.

En general, la incorporación de la evaluación del impacto social a las políticas de I+D no es una realidad sistemática, aunque exista un reconocimiento de la relevancia del impacto social de la acción pública en el ámbito de la ciencia y la tecnología y la necesidad de su medición. En cualquier caso, los intentos de incorporar la evaluación del impacto social se concretan en metodologías de evaluación ex-ante y ex-post, cuyas finalidades son, respectivamente, el apoyo a la toma de decisiones, y la valoración y el aprendizaje (Rip, 2003).

Además, los intentos de incorporar la evaluación del impacto social están sujetos a condicionantes de diferentes tipos entre los cuales pueden citarse los de carácter estructural, relacionados con el comportamiento tangible y la distribución de recursos en las organizaciones involucradas en la integración de la evaluación del impacto social; los condicionantes culturales que incluyen factores actitudinales y valores de los agentes implicados; epistemológicos, vinculados a la fundamentación y definición de conceptos, clasificaciones y procedimientos a utilizar en la integración

en la cohesión económica, social y territorial” con "la consolidación del conocimiento y la innovación como impulsores de nuestro crecimiento futuro".

${ }^{5}$ Real Decreto 1823/2011, de 21 de diciembre, por el que se reestructuran los departamentos ministeriales. BOE, núm. 307, de 22 de diciembre de 2011. 
de la evaluación; y metodológicos, asociados al funcionamiento de los procesos de evaluación de las políticas de I+D+i (Pedrosa et al., 2007).

\subsection{Los estudios de Ciencia, Tecnología y Sociedad}

Los estudios del campo denominado Ciencia, Tecnología y Sociedad (CTS) operan como un área científica que proporciona fundamentación teórica y epistemológica a las prácticas de evaluación del impacto social de la tecnología.

No hay que olvidar que la convicción sobre la necesidad de generar una regulación social de las prioridades y la configuración de la investigación tecnológica no es algo que haya sido siempre evidente. Tradicionalmente, el pensamiento dominante entendía las actividades de $\mathrm{I}+\mathrm{D}$ e innovación tecnológica como un mercado no regulado, en el cual la ciudadanía debía adaptarse a los desarrollos que se originaban por el libre concurso del intelecto, sin que fuera ni necesario ni recomendable las interferencias en este proceso.

Esta idea de una tecnología generada sin vinculación con el medio social se ve cuestionada a partir de la década de 1960 por varias tradiciones de pensamiento, siendo la corriente llamada Ciencia, Tecnología y Sociedad la que mayor impacto ha tenido en esta materia. Por otra parte, los movimientos sociales de carácter reivindicativo, como el antinuclear y los primeros desarrollos del movimiento ecologista, también ayudaron a cuestionar esta visión aproblemática de la tecnología (Jasanoff y Kim, 2009).

Los estudios de Ciencia, Tecnología y Sociedad se originaron en Estados Unidos y Europa a lo largo de la década de 1970 como consecuencia de la acumulación de evidencias sobre las limitaciones del desarrollo científico y tecnológico y el desarrollo de nuevas corrientes de investigación empírica en filosofía y sociología, que planteaban la necesidad de revisar las relaciones entre el mundo científicotecnológico y la sociedad (López Cerezo, 1998).

Estos estudios se centran en la dimensión social de la ciencia y la tecnología, asumiendo como objeto de análisis tanto los factores sociales que influyen en el cambio científico-tecnológico, como las consecuencias sociales y ambientales del cambio tecnológico (González García et al., 1996).

En el caso español, autores de muy variada procedencia han realizado aportaciones al campo CTS desde la década de 1990, alineándose en mayor medida en la tradición europea centrada, principalmente, en los procesos de análisis del cambio científico-tecnológico ${ }^{6}$.

${ }^{6}$ En los estudios CTS a escala internacional pueden distinguirse, en términos generales, dos tradiciones, aunque ambas presentan una alta heterogeneidad. Una de ellas sería la tradición americana centrada en la identificación de los efectos sociales de las tecnologías, 
Gran parte del pensamiento de esta corriente queda de manifiesto en el razonamiento recogido bajo el nombre de Silogismo CTS (González García et al., 1996).

- En primer lugar, el desarrollo científico-tecnológico depende no sólo de la dinámica y evolución interna de la propia ciencia o tecnología, sino que es consecuencia de factores culturales, políticos, económicos, etc. que intervienen en su desarrollo (sociedad modelando la ciencia y la tecnología).

- El cambio científico y tecnológico tiene importantes efectos en las formas de la vida, la sociedad y la biosfera (ciencia y tecnología modelando la sociedad y la naturaleza).

- Las sociedades contemporáneas comparten un compromiso democrático básico, en el sentido de admitir la participación de los diferentes grupos sociales y asumir el diálogo como forma de relación social.

De las premisas anteriores, se concluye que es importante promover la evaluación y el control social del desarrollo científico y tecnológico. Esto significa proporcionar las bases educativas para una mayor participación social y también crear los mecanismos institucionales que hagan posible tal participación.

Ejemplos para el caso español en los que se profundiza en las interacciones entre los elementos tecnológicos y las dinámicas sociales pueden encontrarse en estudios que abordan controversias públicas como son: el llevado a cabo por Aibar y Bijker (1997), dedicado al desarrollo urbano de la ciudad de Barcelona; el realizado por López Cerezo y González García (2002), que analiza las plantaciones forestales en Asturias; o el elaborado por Acero et al. (2011), referido a la cuestión del agua en Cataluña.

De cara al desarrollo de la evaluación del impacto social de proyectos tecnológicos, el pensamiento CTS tiene valor por su componente legitimador, que justifica la necesidad de incorporar aspectos sociales al análisis de la tecnológica y, también, por articular metodologías que permitan dicho análisis. Este planteamiento fundamenta trabajos como el realizado por la Fundación CARTIF donde se recoge una guía para la evaluación del impacto social de proyectos de $\mathrm{I}+\mathrm{D}+\mathrm{i}$ realizados por centros tecnológicos (Moñux Chércoles et al., 2003).

Entre los aprendizajes generados dentro del ámbito CTS destacan: la posibilidad de incluir distintas modalidades de participación pública en el diseño y control del desarrollo tecnológico (López Cerezo et al., 1998); el interés de distinguir diferentes participantes en los procesos de evaluación y poner de manifiesto la importancia de variables vinculadas con el contexto territorial (Aceros et al., 2011); el interés de su aproximación socio-histórica al abordar controversias tecnológicas (Aibar y Bijker, 1997); o la necesidad de avanzar en el desarrollo de indicadores de impacto social (López Cerezo y Luján, 2002).

mientras que la otra, la europea, tiende a orientarse al análisis de los procesos de cambio científico-tecnológico. 


\subsection{El ámbito de la evaluación del impacto ambiental}

La evaluación del impacto ambiental (EIA) es un campo que surge a finales de la década de 1970 en Estados Unidos como respuesta a la necesidad de atender la creciente preocupación de la población por la calidad del medio ambiente ante las grandes inversiones en infraestructuras que se estaban planificando en ese momento. El Congreso de los Estados Unidos publicó en 1969 la Ley Nacional de Política Ambiental (National Environmental Policy Act, NEPA) con el objetivo de fomentar la protección del medio ambiente, entendido como el entorno físico y su población.

A partir de este momento, el uso de la evaluación del impacto ambiental se generaliza a nivel internacional, llegando en la actualidad, a constituir uno de los trámites administrativos básicos para la aprobación de proyectos de muy diversos ámbitos.

En el marco europeo, este tipo de evaluación se empezó a regular a mediados de la década de 1980 y ha sido adoptada progresivamente por todos los países miembros de la Unión Europea. La irrupción de la evaluación del impacto ambiental se produce con la Directiva "Evaluación del Impacto Medioambiental" aprobada por la Comisión Europea en 1985, que asumía como finalidad evitar los daños que puede tener un proyecto en el medio ambiente, proteger la salud humana, contribuir mediante un mejor entorno a la calidad de vida, velar por el mantenimiento de la diversidad de especies y conservar la capacidad de reproducción del sistema como recurso fundamental de la vida. Por tanto, esta Directiva tenía un planteamiento más preventivo que correctivo. Este segundo aspecto de la evaluación del impacto ambiental se integró posteriormente a través de la Directiva 97/11/CE, que incluye la evaluación de las repercusiones de los proyectos públicos y privados sobre el medio ambiente.

España no se ha quedado atrás en este ámbito y ha incorporado y desarrollado paulatinamente una legislación medioambiental propia. A partir de la legislación nacional, la mayoría de las Comunidades Autónomas han desarrollado e implantado leyes autonómicas que regulan la evaluación del impacto ambiental.

De forma paralela al desarrollo legislativo, se ha incrementado el interés por este tema, lo que se refleja en el creciente volumen de trabajos publicados. El campo de análisis de estos trabajos es variado, incluyendo aspectos como los impactos de proyectos de infraestructuras en el medio ambiente, la relación entre impacto ambiental y el desarrollo sostenible, manuales de evaluación para ingenieros y arquitectos o la evaluación ambiental de los planes urbanísticos y de ordenación del territorio. En 1993 se crea la Asociación Española de Evaluación del Impacto Ambiental.

La evaluación del impacto es una parte importante del proceso de evaluación ambiental, que se centra en la previsión y corrección de los efectos de acciones previstas con respecto al medio ambiente. Otro pilar importante es la evaluación ambiental estratégica, cuyo principal objetivo es la valoración de los programas, planes o políticas de medio ambiente. 
Respecto al tema que nos ocupa, hay que señalar que, inicialmente, la preocupación por el impacto social surge como un anexo en la evaluación del impacto ambiental pero, en los últimos años, sobre todo en el contexto internacional, tiende a independizarse con una identidad propia. En el caso estadounidense, a partir de la década de 1970, se incrementa la necesidad de comprender mejor las consecuencias sociales de los proyectos, los programas y las políticas. Como respuesta a esta necesidad, un grupo de científicos sociales crea el Interorganizational Committee on Guidelines Principles for Social Impact Assessment con la finalidad de determinar y establecer una serie de principios que puedan servir de guía para las distintas instituciones e individuos con interés en cumplir sus obligaciones con la legislación medioambiental vigente. Así, y de forma progresiva, la evaluación del impacto social se ha convertido en una herramienta independiente.

También en el contexto de la EIA, pero con un desarrollo metodológico desligado de ésta, se empieza a utilizar la técnica del análisis de ciclo de vida (Life Cycling Assesment, LCA), que se aplica para conocer los potenciales impactos medioambientales de ciertos productos, pero desde una perspectiva más amplia que recoge todas las etapas de su vida: la obtención de las materias primas, la producción, el uso, el reciclado y el descarte definitivo (Manuilova et al., 2009; Tukker, 2000).

Esta técnica ha padecido algunas asimetrías en la profundidad con la que describen los tres pilares de la sostenibilidad (económico, medioambiental y social), desarrollándose posteriormente una línea de trabajo orientada específicamente al análisis del ciclo de vida social (Social Life Cycle Assessment, SLCA) (Griesshammer et al., 2006). Esta alternativa se orienta primordialmente a identificar y evaluar los potenciales impactos socio-económicos negativos y positivos de los productos a lo largo de su ciclo de vida. Labuschagne y Brent (2006 y 2008) definen un conjunto de criterios y subcriterios para medir el impacto social de iniciativas operativas de proyectos y tecnologías de la industria de procesos; Dreyer et al. (2010) han desarrollado un marco de trabajo para la incorporación del pilar social en el análisis de ciclo de vida social en el sector manufacturero; y Lehman et al. (2011) han revisado los impactos sociales identificados en dos proyectos vinculados a la gestión del agua.

De manera general, en el marco español los aspectos sociales son poco tratados en la EIA. Así, Pardo Buendía (2002) analiza la presencia de los aspectos sociales en las evaluaciones del impacto ambiental de embalses, carreteras y autovías, concluyendo que estas evaluaciones están preferentemente orientadas a determinar los efectos negativos, desplazando los efectos positivos, lo que provoca que no pueda realizarse una evaluación pertinente y completa del impacto social en su complejidad. La autora propone medidas para integrar los aspectos sociales en la EIA; entre ellas, la realización de una evaluación ex-ante del impacto social del proyecto, permitiendo así plantear distintas alternativas a los posibles efectos; otra medida sería la realización de estudios de aceptación social del proyecto, a través de procesos de participación pública.

De manera similar, Cantó y Riera (2003) revisan 464 informes de impacto ambiental en Cataluña y detectan una escasa presencia de la parte social y económica 
en dichos estudios; más del 20\% de los estudios revisados no contemplan en absoluto los aspectos socioeconómicos. En general, se da mayor importancia al medio natural y hay una evidente falta de análisis de dinámicas sociales.

En todo caso, los diagnósticos parecen insistir en la necesidad de que la perspectiva ambiental se amplíe con la incorporación de las dimensiones sociales. Un problema especialmente grave se encuentra en las distintas lógicas de los juicios de valor sociales y ambientales, con un marcado incremento de la complejidad en el caso de lo social. De esta manera, es previsible que para incorporar la dimensión social en los aspectos ambientales sean necesarios unos avances metodológicos más profundos que la mera inclusión de unos ítems en un listado de variables.

Un condicionante a considerar en este proceso de integración de los aspectos sociales se deriva del hecho de que la evaluación ambiental ha constituido un grupo profesional bien estructurado, con titulaciones y organizaciones propias, como la Asociación Española de Evaluación del impacto Ambiental, el Congreso Nacional de Evaluación del impacto Ambiental y los Másteres Universitarios en Ingeniería Ambiental de las Universidades de Valencia, Sevilla o Politécnica de Madrid. Todo este entramado profesional, que expresa el dinamismo de la evaluación del impacto ambiental, incrementa la probabilidad de que este colectivo postule la inclusión de la dimensión social dentro del campo ambiental porque así aumentaría su mercado profesional. Este hecho puede llevar a que la dimensión social sea asumida como un añadido, como un suplemento al aspecto ambiental, circunstancia que, unida a que gran parte de los evaluadores ambientales en España tienen formación centrada en las ciencias naturales, dificulta el que los aspectos sociales tengan un papel central. De hecho, en la mayoría de los informes de Evaluación del Impacto Ambiental realizados y analizados la dimensión social tiene un tratamiento claramente secundario. En el estudio realizado por Cantó y Riera (2003) se subraya que, en los casos en los que se incluye un apartado "Medio Socioeconómico" en los informes, cada autor engloba en él aspectos distintos, sin haberse detectado una pauta común: unos autores incluyen sólo aspectos demográficos; otros autores añaden temas de planeamiento urbanístico, situación administrativa, sectores de actividad económica, infraestructuras y servicios, niveles de instrucción de la población, patrimonio arqueológico y cultural, y paisaje.

Sin embargo, y desde el punto de vista de la evaluación del impacto social de proyectos tecnológicos, las herramientas y la experiencia acumulada en este terreno son un referente de utilidad, como muestra el interés en el uso del análisis de ciclo de vida por parte de empresas tecnológicas (Groves et al., 2011).

\subsection{El análisis del impacto de los proyectos de cooperación para el desarrollo}

Otro ámbito especialmente proclive al análisis de los efectos sociales lo constituye los proyectos de cooperación internacional al desarrollo, que persiguen la promoción de las personas y comunidades que se encuentran en una situación de margina- 
ción o pobreza, buscando su desarrollo tanto desde un punto de vista económico como social o medioambiental.

En muchos de estos proyectos, las cuestiones tecnológicas son relevantes, no tanto por lo avanzado de su contenido, sino por las significativas diferencias que existen entre la tecnología utilizada en el proyecto y la tecnología que hasta ese momento caracterizaba la comunidad afectada.

Para el desarrollo de metodologías de evaluación del impacto social de las actividades tecnológicas, la experiencia de los proyectos de cooperación con contenido tecnológico suponen una aportación interesante y valiosa por tres razones: en primer lugar, por la relevancia que se da a las cuestiones sociales en este tipo de actuaciones, en segundo lugar, por la aplicación de un enfoque metodológico aplicable a proyectos y, en tercer y último lugar, por la importancia concedida en estas prácticas evaluativas al análisis del contexto en el que se utiliza la tecnología. Precisamente por el carácter social de su finalidad, esta área es pionera en el análisis del impacto social centrado en proyectos (Roche, 2004).

La evaluación sistemática de los proyectos de desarrollo se concibió y formalizó en la década de 1960 debido a que las agencias donantes buscaban determinar de forma objetiva, profesional e independiente el desempeño y los resultados de las intervenciones (Dale, 2004). Según la organización NONIE ${ }^{7}$, las dos últimas décadas han visto un incremento en la tarea evaluadora (seguimiento/monitorización y evaluación) orientada a los resultados finales que la intervención tiene en el bienestar de individuos y comunidades.

Dentro de esta amplia área de trabajo, las aportaciones realizadas por diferentes actores del mundo de la cooperación al desarrollo, con o sin carácter gubernamen$\mathrm{tal}^{8}$, han permitido perfilar y diferenciar la evaluación del impacto de otras formas de evaluación.

Desde el punto de vista del Banco Mundial, esta evaluación se diferencia de otras semejantes por su especial atención hacia los cambios en el bienestar de los individuos que pueden atribuirse a políticas, programas o proyectos particulares (Gertler et al., 2011). Consecuentemente, responde a preguntas de tipo causa-efecto atribuibles directamente a la intervención.

${ }^{7}$ NONIE es una Red de Redes para Evaluación de Impacto cuyo objetivo es promover la evaluación de impacto de calidad. La red está comprendida por las siguientes organizaciones: Red de Evaluación del Desarrollo del Comité de Asistencia al Desarrollo y de la OCDE, Grupo de Evaluación de las Naciones Unidas (UNEG), el Grupo de Evaluación de la Cooperación (ECG) y la Organización Internacional para Cooperación en Evaluación (IOCE).

${ }^{8}$ La cooperación al desarrollo tiene cada vez más un marcado carácter multilateral con la intermediación de distintas organismos gubernamentales como por ejemplo, el Banco Mundial, el PNUD, el Fondo Monetario Internacional o la Oficina de Cooperación de la Unión Europea. Junto a estos organismos existe otros agentes significativos como son las Organizaciones No Gubernamentales para el Desarrollo (ONGD), por ejemplo, OXFAM, Save the Children o CARE USA. 
Esta misma institución pone de manifiesto dos diferentes enfoques en la evaluación del impacto, distinguiendo entre una evaluación del impacto prospectiva (exante) anterior a la intervención y otra retrospectiva (ex-post) posterior a la intervención, considerando de mayor utilidad la primera. En general, la evaluación del impacto aparece en las primeras y últimas fases en el desarrollo de los proyectos ${ }^{9}$.

El impacto puede ser entendido como uno los criterios de evaluación de la cooperación al desarrollo utilizados por la Organización para la Cooperación y el Desarrollo Económico (OECD DAC Network, 2010) ${ }^{10}$, que analiza el cambio generado por la intervención en los ámbitos económicos, sociales y medioambientales, ya sea positivo o negativo, directo o indirecto, intencionado o no intencionado.

Por su parte, las organizaciones no gubernamentales para el desarrollo (ONGD) detectan una gran dificultad para determinar el impacto real que su actividad tiene en sus potenciales beneficiarios e incluso estudios encargados por ONGD donantes indican una falta de evidencias fiables del impacto de sus proyectos y programas (Riddell et al., 1997; Oakley et al., 1998). Esta realidad se justifica por las dificultades de tipo teórico, financiero, de articulación y metodológicas que existen a la hora de realizar una evaluación del impacto (Rodríguez-Carmona Velasco, 2002).

A pesar de las limitaciones detectadas en la función de evaluación de las ONGDs, la evaluación del impacto social de las intervenciones ha ido creciendo en número, importancia y calidad (Riddell et al., 1997). Las ONGDs líderes, en paralelo con las agencias de cooperación, han realizado un gran esfuerzo por construir sistemas y capacidad de evaluación (Karan, 2009). Por ejemplo, OXFAM ha trabajado la evaluación del impacto durante todo el desarrollo del ciclo del proyecto, atendiendo a la diversidad de tipos de proyectos, metodologías, contextos y, por tanto, la diversidad de dificultades, proponiendo una serie de consideraciones a la hora de diseñar el proceso de evaluación (Roche, 2004). Igualmente, OXFAM muestra la dificultad de establecer y manejar la información sobre grupos de control así como los posibles problemas éticos derivados de no colaborar deliberadamente con un grupo que puede precisar de ayuda, lo que supone poner en tela de juicio la idea propuesta por las principales agencias de desarrollo de llevar a cabo un análisis contrafactual, para poder realizar convenientemente la atribución de los resultados a un proyecto.

Desde el punto de vista español, la labor realizada por el Ministerio de Asuntos Exteriores y Cooperación, a través de la Agencia Española de Cooperación Internacional al Desarrollo (AECID), y por distintas ONGD españolas con proyectos de cooperación en el ámbito de la tecnología (Ingeniería Sin Fronteras, ISF, o el Grupo

${ }^{9}$ Las seis fases reconocidas en el desarrollo de un proyecto: identificación, preparación, evaluación inicial, aprobación, ejecución/terminación y evaluación.

${ }^{10}$ La OCDE considera los siguientes cinco criterios: relevancia, eficacia, eficiencia, impacto y sostenibilidad. 
de Organización, Calidad y Medio Ambiente, GOCMA) permite poder hablar de avances significativos en evaluación del impacto social en este ámbito.

A finales de la década de 1990, el citado Ministerio comenzó a trabajar en una metodología de evaluación de la cooperación española con el propósito de determinar la calidad de la ayuda ofrecida, trabajo que se materializó en 1998 con la publicación de la metodología de evaluación de la cooperación española (Ministerio de Asuntos Exteriores, 1998), revisada y actualizada tres años más tarde (Ministerio de Asuntos Exteriores, 2001) y que, en 2007, dio lugar a un manual de gestión de evaluaciones de cooperación (Ministerio de Asuntos Exteriores y Cooperación, 2007). Estos documentos siguen la línea marcada por la OCDE y, en este sentido, la evaluación del impacto se considera únicamente al final del proyecto y como medida de los efectos de la intervención realizada. Los motivos para la promoción de la evaluación del impacto son similares a los indicados por agencias internacionales: transparencia, rendición de cuentas e información en clave de aprendizaje.

En 2010, se publica el primer informe anual sobre la evaluación en la cooperación española (Ministerio de Asuntos Exteriores y Cooperación, 2010) que, aunque no permite discriminar qué parte de la evaluación realizada por los distintos agentes de la cooperación española se refiere a la evaluación del impacto, sí que muestra datos generales de interés para valorar la expansión de la cultura evaluativa dentro de los actores de la cooperación.

Finalmente, las ONGD españolas con proyectos de cooperación en el ámbito de la tecnología no incorporan de manera sistemática la evaluación del impacto de sus proyectos con una metodología uniforme, pero sí se constata la realización de una evaluación diagnóstica al inicio y al final de proyectos concretos, aunque sin una metodología explícita sobre evaluación del impacto. Por ejemplo, el "Manual de Energía Solar Fotovoltaica y Cooperación al Desarrollo” (Ingeniería sin Fronteras, 1999) incluye una metodología para desarrollar un proyecto que contempla un estudio inicial de la comunidad beneficiaria con variables técnico-sociales como: vida material, organización social, organización doméstica, organización del trabajo, organización espacial y colaboradores locales y, también, un estudio económico y una evaluación final sobre la que se establece una duración mínima de dos años y se propone un análisis pormenorizado similar al realizado en la etapa inicial. Se incluye, además, la necesidad de un comunicador y diálogo con la comunidad receptora. $\mathrm{O}$ el informe sobre el mecanismo de desarrollo limpio elaborado por miembros del Grupo de Organización, Calidad y Medio Ambiente (GOCMA) de la Universidad Politécnica de Madrid (Guijarro et al., 2008), en el que se plantea una metodología para la evaluación del impacto de los proyectos, considerando tanto evaluación ex-ante como evaluación ex-post.

Consecuentemente, es posible considerar al campo de la cooperación al desarrollo como un ámbito innovador en la creación de metodologías de evaluación del impacto social de las actividades tecnológicas por dos razones, por un lado, por su sensibilidad hacia la temática social $\mathrm{y}$, por otro, por el contenido tecnológico de algunas de sus intervenciones, como se refleja en la presencia de ingenieros en esos proyectos. Sin embargo, sus propuestas requerirían un proceso de adaptación para 
poder ser empleadas en el contexto de una sociedad como la española, que presenta aspectos diferenciales respecto a la mayoría de países receptores de la ayuda pública a la cooperación.

\subsection{La Responsabilidad Social Corporativa}

Este epígrafe se dedica a la Responsabilidad Social Corporativa (RSC) ${ }^{11}$, enfoque que a pesar de presentar una orientación generalista, no estrictamente tecnológica, y plantear a las entidades organizativas como objeto de análisis en vez de las actividades o proyectos concretos, puede suponer una aportación de interés a la evaluación del impacto social de proyectos tecnológicos.

Estas aportaciones pueden ser relevantes, por ejemplo, en el caso de empresas que generan tecnología o la usan con intensidad, como ocurre en el sector químico o el de la electrónica.

A diferencia de aportaciones en otros ámbitos como la evaluación de tecnologías o de políticas de I+D e innovación, vinculadas con el sector público, la evaluación de tecnología en el contexto de la RSC se desarrolla en organizaciones mayoritariamente pertenecientes al sector privado, hecho que dificulta contar con información sistemática, salvo para el caso de grandes empresas con políticas de comunicación especialmente transparentes.

El término de RSC se generalizó en el contexto internacional a finales de la década de 1960 de la mano de empresas multinacionales que tenían un creciente interés por conocer los efectos de su actividad sobre las partes interesadas (stakeholders) ${ }^{12}$, la sociedad en su conjunto y el medio ambiente. La incorporación de la responsabilidad social en la gestión de la empresa supuso tomar una postura activa y responsable en torno al impacto de su actividad, buscando la sostenibilidad económica, medioambiental y social (NOKIA, 2012).

En general, el hecho tecnológico no asume un especial protagonismo en las prácticas de RSC incluso en las empresas usuarias y/o generadoras de tecnología. Una excepción lo presentan algunas empresas que manejan tecnologías sujetas a una importante incertidumbre sobre posibles impactos futuros, como es el caso de la nanotecnología o la biotecnología (células madre, organismos modificados genéticamente...). En cualquier caso, la toma de conciencia por parte de las empresas sobre su responsabilidad frente a la sociedad ha llevado a la aparición de actuacio-

${ }^{11}$ En el caso de España, se utiliza de manera indistinta los términos de responsabilidad social corporativa y responsabilidad social empresarial.

12 El concepto de stakeholder, introducido por Freeman en el año 1984 en su obra Strategic management: a stakeholder approach, constituye un elemento esencial en los procesos de evaluación de impacto al permitir incorporar de manera sistemática a todas las partes afectadas. 
nes proactivas respecto al impacto social de la tecnología por su parte, destacando, para el ámbito europeo la metodología SEEBalance (Schmidt et al., 2004) o el proyecto PROSA (Griesshammer et al., 2007) ${ }^{13}$.

La primera desarrolla una herramienta que mide los aspectos sociales de la sostenibilidad y los añade al análisis de eficiencia ecológica ya desarrollado por la empresa BASF y, la segunda, diseña un método para estudiar aspectos económicos, sociales y medioambientales a través del ciclo de vida de un producto y su cadena de valor, por ejemplo, para el caso de la producción de un notebook (Manhart y Griesshammer, 2006).

En el caso particular de actividades empresariales con un alto contenido tecnológico, la presencia de la RSC es esencial a la hora de considerar el impacto social de sus actividades, ya que, a pesar de que existe una estricta regulación de obligado cumplimiento en el ámbito nacional y europeo, en ocasiones, la acción pública tiene una limitada capacidad para regular los desarrollos y prácticas tecnológicas de vanguardia. Esta situación es frecuente en sectores que no disponen de información adecuada para legislar en cuestiones sometidas a continuos cambios debido al dinamismo tecnológico. Los actores responsables de ese dinamismo están en buenas condiciones para gobernar el uso responsable de la tecnología y la innovación, anticipando de forma proactiva los impactos de su actividad (Groves et al., 2011).

En España, la RSC empieza a tomar fuerza a finales de la década de 1980 debido, en gran parte, a la necesidad que experimentaban las empresas nacionales que atravesaban procesos de internacionalización de mostrar la responsabilidad de su comportamiento. En este sentido, también fue un importante catalizador del desarrollo de la RSC la aparición de productos financieros éticos, con sus consiguientes problemas de definición y evaluación (Cuesta González y Valor Martínez, 2003).

Como ocurre en el contexto internacional, dentro de las empresas que generan o usan tecnología con intensidad, únicamente pueden encontrarse trabajos sobre la responsabilidad social para el caso de grandes empresas. Estos trabajos tienen un enfoque generalista, y se evalúan las actuaciones de extensión social de la empresa más que las consecuencias reales que la tecnología tiene sobre los ciudadanos (Sacristán y Gómez, 2009).

A diferencia de lo que sucede en otros países europeos, los autores del presente trabajo no han localizado ninguna práctica de evaluación del impacto social de la tecnología en el marco de actuaciones de RSC, aunque no puede concluirse de este hecho el que no se hayan realizado este tipo de prácticas, puesto que el mundo de la empresa se caracteriza por operar como una "caja negra” en la cual la información

${ }^{13}$ La metodología SEEBalance es el resultado de un trabajo conjunto entre la empresa BASF, la Universidad de Karlsruhe y el Instituto para la Ecología Aplicada (Öko-Institute e.V.). La herramienta PROSA ha sido desarrollada por el Instituto para la Ecología Aplicada en colaboración con otras empresas. PROSA sirve también para el análisis de tecnologías y grandes proyectos de infraestructuras. 
que se ofrece es escasa debido a la necesidad de mantener confidencialidad sobre sus operaciones como fuente de su ventaja competitiva.

La RSC es una orientación claramente impulsada por instancias públicas tanto europeas como españolas. Para las primeras puede citarse el Libro Verde "Fomentar un marco europeo para la responsabilidad social de las empresas" elaborado por la Comisión Europea en 2001, la resolución del Parlamento Europeo, de 13 de marzo de 2007, sobre la responsabilidad social de las empresas, o la comunicación "Una estrategia renovada 2011-2014 para la responsabilidad social corporativa" de la Comisión Europea (European Comisión, 2011). Para las segundas, destaca el apoyo al Observatorio de la Responsabilidad Social Corporativa, creado en 2004, y la creación en 2008 del Consejo Estatal de Responsabilidad Social de las Empresas.

La línea de trabajo de la RSC reconoce que las empresas tienen un significativo impacto en la sociedad. Para las empresas que usan o generan tecnología este impacto presenta especificidades a la hora de su análisis y gestión, como muestran el desarrollo específico llevado a cabo por empresas e institutos tecnológicos.

Durante las últimas décadas, fruto del intento de gestionar dicha responsabilidad, se ha materializado un amplio catálogo de actividades e instrumentos, con la colaboración de actores públicos y privados con y sin afán de lucro, como son los códigos éticos y de conducta, sistemas de gestión específicos o informes de responsabilidad social. Entre los distintos referenciales y estándares relativos a la RSC reconocidos actualmente en España pueden citarse: la guía ISO26000 sobre Responsabilidad Social de la International Organization for Standardization, el estándar de certificación SA8000 sobre prácticas socialmente aceptables de la organización Social Accountability International, la norma SGE21 de certificación en gestión ética y responsabilidad social de la organización Forética, la norma UNE165010 "Ética. Sistema de gestión de la responsabilidad social en la empresa" desarrollada por AENOR o la especificación RS10 de AENOR sobre Sistema de Gestión de la Responsabilidad Social.

Toda esta experiencia acumulada es útil para la evaluación del impacto social de proyectos tecnológicos. Por ejemplo, el establecimiento de principios o las dimensiones clave de la responsabilidad social de la ISO26000 o la SA8000 pueden constituir un marco de trabajo de dicha evaluación.

Al mismo tiempo, la evaluación del impacto social de proyectos tecnológicos puede ser una herramienta para orientar las actuaciones de las empresas en el ámbito de la RSC. Así, a la hora de poder fijar el marco de referencia de su responsabilidad social, las empresas deben ocuparse de todo el ciclo de vida de los productos generados, tal como propone la evaluación de proyectos. Con esa finalidad, pueden acudir a técnicas que permitan estudiar el impacto en las diferentes etapas del ciclo de vida, como el ya mencionado análisis del ciclo de vida social. 


\section{Reflexiones sobre la evaluación del impacto social de actividades tecnológi- cas en España}

El apartado precedente, que recoge aportaciones relevantes para la evaluación del impacto social de la tecnología en distintos ámbitos, revela la existencia de una diversidad de enfoques en la forma de entender y evaluar el impacto social de la tecnología, generando un conjunto heterogéneo de prácticas que sintetiza la tabla 2. El estudio de estas prácticas permite asegurar que en la actualidad la evaluación del impacto social de actividades y proyectos tecnológicos no constituye en España una práctica identificable, pudiéndose hablar, fundamentalmente, de contribuciones relevantes en áreas afines. Sin duda, esta diversidad puede enriquecer el análisis del impacto social de las actividades tecnológicas, pero para ello es fundamental que se realice una revisión crítica de estos enfoques considerando sus potencialidades y, en su caso, sus limitaciones.

Un primer aspecto a considerar, tal y como ya se presentó en el apartado anterior y se recoge en la Tabla 1, es el relacionado con el objeto de evaluación y su orientación, que permite distinguir tres ámbitos de análisis con aportaciones diferenciadas.

El primer ámbito realiza aportaciones en el contexto de un análisis de las consecuencias de los hechos tecnológicos con un enfoque más amplio que el de proyectos, como es el caso de los estudios CTS o la evaluación de tecnologías y de políticas de $\mathrm{I}+\mathrm{D}+\mathrm{i}$. Entre estas aportaciones destaca el desarrollo de metodologías que se ha realizado en el campo de la evaluación de tecnologías y en los estudios CTS, donde se hace hincapié en la necesidad de la participación pública en las evaluaciones. Además, se pone de manifiesto la existencia de condicionantes de diversa índole cuando se evalúan controversias públicas sobre cuestiones tecnológicas o políticas de I+D+i. También es relevante el carácter legitimador de la evaluación del impacto social que ofrecen los estudios CTS y la sistematización de impactos sociales que realiza la evaluación de tecnologías.

El segundo ámbito realiza aportaciones relacionadas con el análisis de impacto de proyectos, como es el caso de la evaluación del impacto ambiental o la evaluación de proyectos de cooperación al desarrollo. Entre sus aportaciones destaca el desarrollo de metodologías de evaluación para proyectos que pueden ser transferibles a actuaciones que persigan evaluar el impacto social de proyectos tecnológicos. De hecho, la vertiente social ya forma parte de estas evaluaciones, aunque no esté plenamente consolidada, ya que el interés por la dimensión social es reciente y está en proceso de incorporación de manera explícita y detallada. Además, destaca la relevancia del contexto para la identificación de stakeholders y la definición de los impactos sociales de los proyectos, lo que condiciona el proceso de evaluación.

El tercer ámbito, que incluye únicamente la RSC, recoge aportaciones relativas a la necesidad de conocer y mejorar el impacto social de las actividades tecnológicas en el contexto de unas empresas preocupadas por conocer los efectos de su actividad sobre la sociedad. Distintos organismos de estandarización y certificación que trabajan en temas vinculados a la RSC han desarrollado metodologías de identificación y gestión de distintos impactos sociales. 
Esta extensión hacia la dimensión social presenta algunos problemas asociados. Por ejemplo:

- La lógica que subyace en el proceso de evaluación del impacto en el ámbito medioambiental es diferente a la que requiere una evaluación del impacto social. A modo de ejemplo, en la dimensión social los juicios de valor suelen ser más conflictivos, existe más dificultad para establecer una atribución clara de los impactos y existen dinámicas de desplazamiento de los efectos.

- Los intereses existentes y la experiencia y formación acumuladas por los responsables de la evaluación de corte medioambiental hasta este momento condiciona y limita la posibilidad de llevar a cabo cambios relevantes en la forma de valorar los proyectos. Este hecho queda reflejado, por ejemplo, en el análisis de Cantó y Riera (2003) anteriormente citado, en el que se destaca la escasez de equipos pluridisciplinarios y la ausencia casi total de profesionales de las ciencias sociales en los equipos de redacción de los informes realizados. La mayoría de los trabajos fueron realizados por ingenieros de caminos, canales y puertos, seguidos por arquitectos.

Un segundo aspecto de las contribuciones consideradas, que puede condicionar su utilización en la evaluación del impacto social de proyectos tecnológicos, es el diferente nivel de institucionalización y desarrollo de las distintas tradiciones. Mientras algunas de ellas se encuentran en sus primeras fases de desarrollo, existen otras, como la evaluación del impacto ambiental, que cuentan con una amplia implantación, con una regulación legal, estandarización metodológica y con un cuerpo profesional con una formación reglada.

Estas diferencias en los niveles de institucionalización de las distintas tradiciones puede ser un importante condicionante del formato futuro que asuma la evaluación del impacto social de proyectos tecnológicos, puesto que en función de la fuerza de los diferentes colectivos profesionales o de la mayor o menor eficacia organizativa de las entidades promotoras, determinados planteamientos metodológicos acabarán triunfando e imponiéndose sobre otros.

Esta realidad puede condicionar la forma en que se incorpore la perspectiva social y la influencia que esta línea de trabajo pueda llegar a tener en el desarrollo de los procesos de evaluación del impacto de la tecnología.

Un tercer aspecto a considerar es el dispar grado de implicación del sector público en los distintos ámbitos, hecho que puede condicionar la velocidad de desarrollo de las distintas líneas de trabajo y su contribución a la evaluación del impacto social de los proyectos tecnológicos. Por un lado, el sector público español ha mostrado interés en relación al análisis de impacto en temas relacionados con el medio ambiente, y en menor medida y más recientemente, con la ayuda al desarrollo y con la RSC, lo que ha fomentado el desarrollo de metodologías comúnmente aceptadas. Por otro lado, la evaluación de tecnologías no ha estado presente tan activamente en la agenda política española y, por tanto, no ha existido el efecto demostración en este último caso. 
Tabla 2. Aportaciones para la evaluación del impacto social de la tecnología de diversas líneas de trabajo vinculadas

\begin{tabular}{|c|c|c|c|c|c|c|}
\hline 产 촐 & 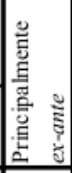 & 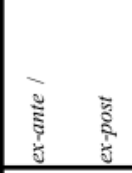 & 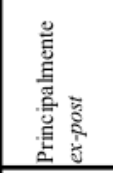 & 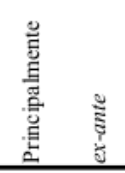 & 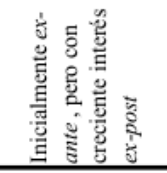 & 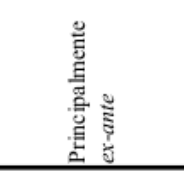 \\
\hline 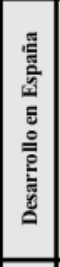 & 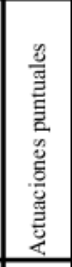 & 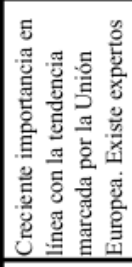 & 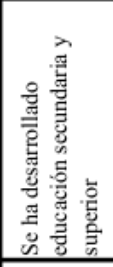 & 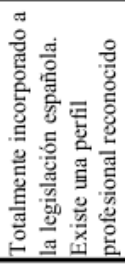 & 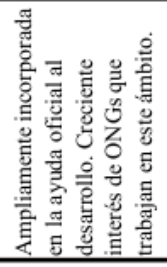 & 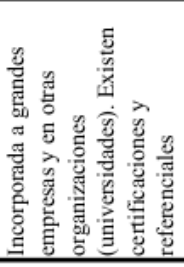 \\
\hline 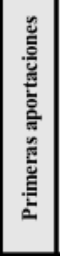 & 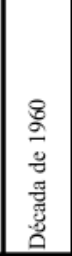 & 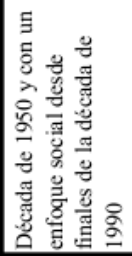 & 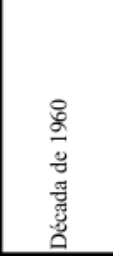 & 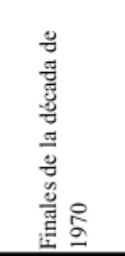 & 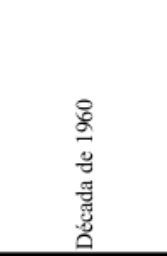 & 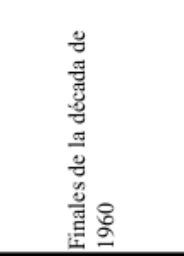 \\
\hline 递 & 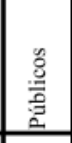 & 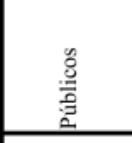 & 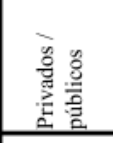 & 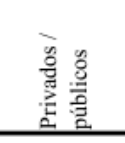 & 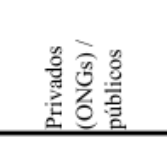 & 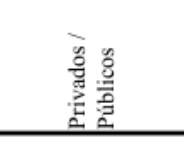 \\
\hline 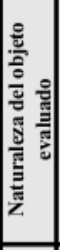 & 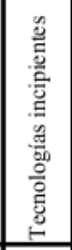 & 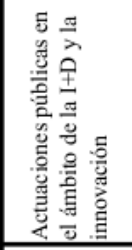 & 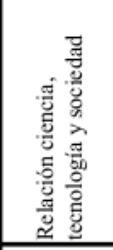 & 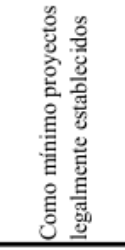 & 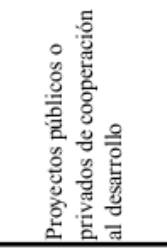 & 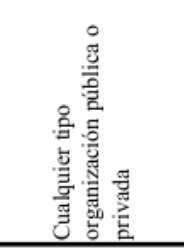 \\
\hline 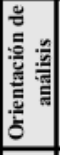 & 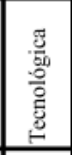 & 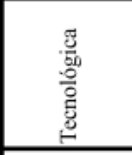 & 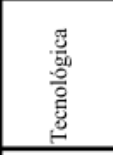 & $\begin{array}{l}\text { 范 } \\
\text { 总 } \\
\end{array}$ & 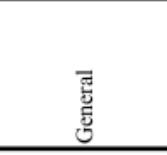 & 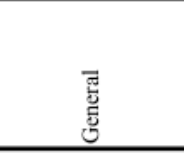 \\
\hline 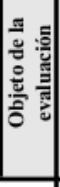 & 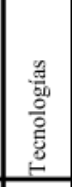 & 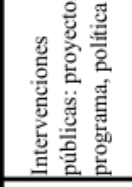 & 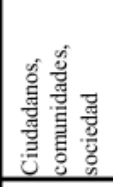 & 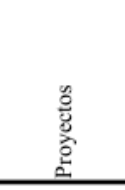 & 总 & 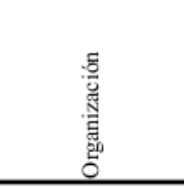 \\
\hline & 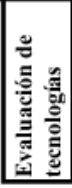 & 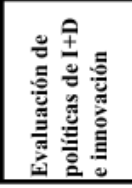 & 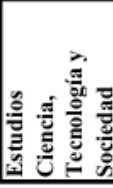 & 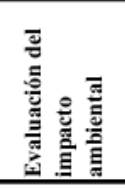 & 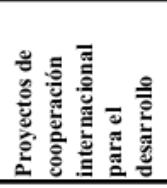 & 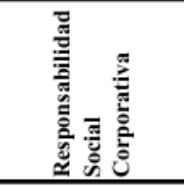 \\
\hline
\end{tabular}

Fuente: elaboración propia. 
Los procesos de institucionalización de determinadas prácticas en evaluación del impacto ambiental o en RSC pueden ser un elemento impulsor importante para el desarrollo futuro de procesos de evaluación del impacto social de los proyectos tecnológicos, con la presencia de entidades certificadoras, referenciales de comportamiento, una formación reglada reconocida y unos profesionales acreditados. Sin embargo, esta dinámica puede llegar a tener efectos secundarios no deseables, como son la aparición de una obsesión credencialista, que lleve a una dinámica de burocratización y mercantilización orientada a la obtención de certificaciones con reducido contenido.

Un último elemento a considerar es el diferente enfoque temporal de las tradiciones de evaluación que se han presentado en este trabajo. La evaluación del impacto social de proyectos tecnológicos puede aplicarse en diversos momentos del ciclo de vida de los proyectos, de manera que en cada uno de esos momentos puede aprovechar las aportaciones y conocimiento acumulado por las tradiciones citadas. Por poner un ejemplo, en el desarrollo de una evaluación del impacto social ex-ante de un proyecto tecnológico puede ser muy relevante la experiencia de la evaluación del impacto ambiental, mientras que en la evaluación con un enfoque temporal más amplio, puede ser interesante el caso de las políticas de I+D+i, cuya metodología se extiende a lo largo de todas las etapas del ciclo de vida de las políticas públicas. También puede hacer uso de las metodologías de evaluación ex-post que se utilizan en evaluación de proyectos de cooperación o de intervenciones públicas en I+D+i y que está orientada a procesos de aprendizaje.

\section{Conclusiones y consideraciones para el futuro}

A modo de conclusión puede afirmarse que, en general, España no cuenta con una práctica consolidada de rendición de cuentas a los ciudadanos sobre la acción pública o empresarial y, en particular, puede reconocerse que la evaluación del impacto social de actividades y proyectos tecnológicos en España no constituye una línea de investigación claramente identificable dentro de la comunidad científica.

Puede afirmarse, al mismo tiempo, que al ampliar, por un lado, el ámbito de estudio considerando una orientación generalista del análisis, no solo tecnológica, y, por otro lado, el objeto de trabajo, yendo más allá de un proyecto o actividad concreta, existen campos afines que presentan conocimientos y experiencias próximas a la evaluación del impacto social de actividades y proyectos tecnológicos, siendo una fuente de información útil para dicha evaluación. En el caso de España, el volumen de las aportaciones en los distintos campos es desigual, destacando positivamente los estudios CTS y los proyectos de cooperación al desarrollo, existiendo aportaciones puntuales en la Evaluación de Tecnologías y en la EIA y siendo mucho menores las aportaciones en el caso de la evaluación de políticas de I+D+i y la RSC. En líneas generales, se detecta que en algunos de esos campos las aportaciones a nivel internacional son significativamente superiores a las que existen en 
España, como ocurre en la evaluación de tecnologías o la evaluación del impacto ambiental.

Las aportaciones a la evaluación del impacto social de actividades tecnológicas de los campos analizados son heterogéneas y complementarias en aspectos como metodologías, herramientas, identificación de impactos sociales e indicadores asociados, factores condicionantes o carácter legitimador de la evaluación del impacto social de la tecnología. Estas aportaciones tienen un distinto enfoque temporal, siendo predominante el ex-ante para la evaluación de tecnologías, la evaluación del impacto ambiental y la RSC, mientras que el resto se extiende también a enfoques in-itinere y ex-post.

Además, esas aportaciones tienen un distinto grado de institucionalización, siendo más intensa para la evaluación del impacto ambiental, seguida a distancia por la RSC y la evaluación de proyectos de cooperación. Este hecho puede condicionar la influencia de las distintas líneas de trabajo en el desarrollo de los procesos de evaluación del impacto social de proyectos tecnológicos. Esta institucionalización se debe en parte a la presencia del sector público, por su papel como regulador y financiador de actividades con impacto social, lo que muestra cómo este sector puede ser un actor relevante en dicho desarrollo.

A partir de las reflexiones anteriores, y en opinión de los autores, pueden realizarse algunas consideraciones sobre el futuro de la evaluación del impacto social de los proyectos tecnológicos en España:

En primer lugar, a pesar del escaso nivel de desarrollo actual, consideramos que sería deseable un incremento de su presencia y relevancia futura tanto en la esfera pública como privada, que estaría justificado por el extraordinario peso que la tecnología tiene en las sociedades contemporáneas, como se está haciendo, por ejemplo, en el caso del proyecto europeo PROSUITE (Prospective Sustainability Assessment of Technologies), dedicado al desarrollo de una metodología para la evaluación del impacto sostenible de nuevas tecnologías (PROSUITE PROYECT, 2013) .

El sector público puede tener un papel relevante en su futuro desarrollo, que se justificaría por el creciente interés de vincular las actividades de I+D e innovación con el bienestar y la calidad de vida de los ciudadanos y por la experiencia de este sector en otros campos de la práctica evaluativa.

Es también previsible la incorporación a esa dinámica de otros actores privados como, ONGs, centros de investigación, etc. y cuya participación puede incrementarse como consecuencia de una dinámica social que intenta revisar los valores de la economía partiendo de una orientación que persigue el bien común alejándose de la lógica de los mercados (Felber, 2012).

Y, por último, sería interesante dotar a la evaluación del impacto social de los proyectos tecnológicos de una metodología propia, de carácter interdisciplinar y vinculada a la empleada en otros campos afines. El desarrollo de esta metodología requiere de:

- Debates abiertos, evitando establecer dicotomías insalvables entre metodologías rígidas o plurales o, también, entre opciones participativas o tecnocráticas. 
- Un planteamiento pluralista, pues la opción por referenciales cerrados, auditados por expertos, siguiendo modelos inspirados en las normas de calidad, es un modelo cuya aplicación para el caso de la evaluación del impacto social de la tecnología puede ser, cuando menos, problemática.

- Un sistema de evaluación que fomente el debate social y origine información diversa, evitando la aritmética social. Aunque la idea de presentar una cifra para sintetizar el impacto social de un proyecto tecnológico sería viable y tiene sus ventajas de cara a realizar una comunicación mediática con capacidad para incidir fácilmente en la opinión pública, tiene riesgos de generar arbitrariedad, rigidez y falta de fundamento epistemológico.

En el comienzo del siglo XXI la tecnología juega un importante papel en la economía y la vida social. Dentro de los recursos necesarios para realizar una gobernanza eficaz de esta realidad, la evaluación del impacto social de las actividades y proyectos tecnológicos constituye un instrumento valioso para la gestión y la planificación eficaz de sus efectos.

\section{Agradecimientos}

Este artículo ha sido elaborado en el marco del proyecto de investigación "Políticas de la cultura científica: análisis de las dimensiones políticas y sociales de la cultura científica” (FFI2011-24582), financiado por el Ministerio de Economía y Competitividad de España.

\section{Bibliografía}

Aibar, E. y Bijker, W.E. (1997): "Constructing a City: The Cerda Plan for the Extension of Barcelona". Science, technology, \& human values, 22, 1: 3-30. DOI 10.1177/016224399702200101

Albornoz, M., Estébanez, M.E. y Alfaraz, C. (2005): “Alcances y limitaciones de la noción de impacto social de la ciencia y la tecnología”, Revista CTS, 4: 73-95. Disponible en: http://www.scielo.org.ar/pdf/cts/v2n4/v2n4a05.pdf (Consulta: 22 mayo 2013)

Acero, J.C.; Tirado, F. y Domènech, M. (2011): Percepciones de los ciudadanos catalanes acerca de la participación del público y los expertos en la controversia del agua. Paper, 96, 1: 227-244. Disponible en: http://www.raco.cat/index.php/papers/article/viewFile/229242/310952 (Consulta: 22 mayo 2013)

Banco de España (2012): Balanza de Pagos y Posición de Inversión Internacional de España 2012. Banco de España. Madrid.

Becker, H.A. y Vanclay, F. (2003): The international handbook of social impact assessment. Cheltenham, Edward Elgar. 
Cantó, S., Riera, P. (2003): "La vertiente socioeconómica en los estudios de impacto ambiental”, Ciudad y Territorio. Estudios Territoriales, 138: 539-550.

Cantó-Milà, N. y Lozano, J.M. (2009): "The Spanish discourse on corporate social responsability". Journal of Business Ethics, 87: 157-171.

Chedd, G. (1973): "Emilio Dadario. The godfather of TA", New Scientist, 58, 847: 490-492.

Cuesta González, M. y Valor Martínez C. (2003): "Responsabilidad social de la empresa. Concepto, medición y desarrollo en España”. Boletín económico del ICE, 2755, 7-19. Disponible http://www.revistasice.com/CachePDF/BICE_2755_0719_843B2AFA16833BD45F65BF48332D2587.pdf (Consulta: 22 mayo 2013)

Dale, R. (2004): Evaluating Development Programmes and Projects, New Delhi, Sage Publications.

Decker, M; Ladikas, M. (editores) (2004): Bridges between Science, Society and Policy. Technology Assessment - Methods and Impacts. Springer. Berlin.

Dreyer, L. C., Hauschild, M. Z. y Schierbeck, J. (2010): “Characterisation of social impacts in LCA", The International Journal of Life Cycle Assessment, 15, 3: 247-259. DOI: 10.1007/s11367-009-0148-7

Estébanez, M.E. (2003): "Impacto social de la ciencia y la tecnología: estrategias para su análisis”. En: Red Iberoamericana de Indicadores de Ciencia y Tecnología (2003): El Estado de la Ciencia 2002. Principales indicadores de la Ciencia y la Tecnología, Buenos Aires, RICYT-CYTED.

Esteves, A.M., Franks, D. y Vanclay, F. (2012): "Social impact assessment: the state of the art". Impact Assessment and Project Appraisal, 30, 1: 34-42. DOI:10.1080/14615517.2012.660356

EURAB, (2005): "Science and Society": An agenda for responsive and responsible European science in FP7. EURAB. Disponible en: http://ec.europa.eu/research/eurab/pdf/eurab_05_035_wg6_final_reportrev_160905.pdf (Consulta: 22 mayo 2013)

European Commission (2011): A renewed EU strategy 2011-14 for Corporate Social Responsibility. COM(2011) 681 final. Brussels, European Commission. Disponible en: http://ec.europa.eu/enterprise/newsroom/cf/_getdocument.cfm?doc_id=70 10 (Consulta: 22 mayo 2013)

Eurostat (2010): Special eurobarometer 340, Science and Technology. Eurostat. Bruselas. Disponible en: http://ec.europa.eu/public_opinion/archives/ebs/ebs_340_en.pdf (Consulta: 22 mayo 2013)

Feber, C. (2012): La economía del bien común, Ediciones Deusto. Bilbao.

FECYT (2006): Carencias y necesidades del Sistema Español de Ciencia y Tecnología. Informe 2005, Madrid, FECYT. Disponible en: http://icono.fecyt.es/informesypublicaciones/Documents/carencias.pdf (Consulta: 22 mayo 2013) 
Fernández Esquinas, M., Díaz Catalán, C. y Ramos Vielba, I. (2011): "Evaluación y política científica en España: el origen y la implantación de las prácticas de evaluación científica en el sistema público de I+D (1978-1994)". En González de la Fe, T. y López Peláez, A. (2011): Innovación, conocimiento científico y cambio social. Ensayos de sociología ibérica de la ciencia y la tecnología. CIS. Madrid: 93-130.

Fundación COTEC (2011): Tecnología e innovación en España. Informe 2011, Madrid, COTEC.

Gertler, P.J., Martinez, S.; Premand, P.; Rawlings, L.B. y Vermeersch C.M.J. (2011): La evaluación del impacto en la práctica. Washington. World Bank Publications.

González García, M.I.; López Cerezo, J.A. y Luján López, J.L. (1996): Ciencia, tecnología y sociedad: una introducción al estudio social de la ciencia y la tecnología, Madrid, Tecnos.

Griesshammer, R., Benoît, C., Dreyer, L.C., Flysjö, A., Manhart, A., Mazijn, B., Méthot, A.L. y Weidema, B. (2006): Feasibility Study: Integration of social aspects into LCA, Freiburg, Öko-Institut e.V. Disponible en: http://www.saiplatform.org/uploads/Library/UNEP-

SETACLifeCycleInitiativeTFonSocialIssues-FeasibilityStudy.pdf (Consulta: 22 mayo 2013)

Griesshammer, R., Buchert, M., Gensch, C.-O., Hochfeld, C., Manhart, A., Reisch, L. y Rüdenauer, I. (2007): PROSA - Product Sustainability Assessment Guideline, Freiburg, Öko-Institut e.V. and Institute for Applied Ecology. Disponible en:

http://www.prosa.org/fileadmin/user_upload/pdf/leitfaden_eng_final_310 507.pdf (Consulta: 22 mayo 2013)

Groves, C.; Frater, L.; Lee, R. y Stokes, E. (2011): "Is there room at the bottom for CSR? Corporate social responsibility and nanotechnology in the UK”. Journal of business ethics, 101: 525-552. DOI: 10.1007/s10551-010-0731-7

Guijarro, A., Lumbreras, J. y Habert, J. (2008): El Mecanismo de Desarrollo Limpio y su contribución al Desarrollo Humano. Análisis de la situación y metodología de evaluación del impacto sobre el desarrollo, Madrid, Intermón Oxfam. Disponible en: http:/www.ongawa.org/wpcontent/uploads/2011/08/INV_MDL.pdf (Consulta: 22 mayo 2013)

Ingeniería sin Fronteras (1999): Energía solar fotovoltaica y cooperación al desarrollo. Cooperación y Tecnología. Ingeniería sin Fronteras y IEPALA. Madrid.

Interorganizational Committee on Principles and Guidelines for Social Impact Assessment (2003): "Principles and guidelines for social impact assessment in the USA". Impact Assessment and Project Appraisal, 21, 3: 231-250. DOI: $10.3152 / 147154603781766293$

Jasanoff, S. y Kim, S.H. (2013): "Sociotechnical imaginaries and national energy policies". $\quad$ Science as culture, 22, 2: 189-196. DOI: 10.1080/09505431.2013.786990 
Jiménez Hernández, E. (2009): Reinterpretando la rendición de cuentas o accountability: diez propuestas para la mejora de la calidad democrática y la eficacia de las políticas públicas en España. Documento de trabajo 145/2009. Fundación alternativas. Barcelona. Disponible en: http://www.falternativas.org/content/download/12503/376439/version/5/fi le/doc145.pdf (Consulta: 22 mayo 2013)

Karan, L. (2009): Evaluation use in non-governmental organization: Unlocking the «Do-Learn-Plan» continuum (Thesis), Medford, Tufts University.

Labuschagne, C. y Brent, A. (2006): "Social Indicators for Sustainable Project and Technology Life Cycle Management in the Process Industry”. The International Journal of Life Cycle Assessment, 11, 1: 3-15. DOI:10.1065/lca2006.01.233

Labuschagne, C. y Brent, A.C. (2008): “An industry perspective of the completeness and relevance of a social assessment framework for project and technology management in the manufacturing sector”. Journal of Cleaner Production, 16, 3: 253-262. DOI: 10.1016/j.jclepro.2006.07.028.

Lehman, A.; Russi, D.; Bala, A.; Finkbeiner, M; y Fullana-i-Palmer, P. (2011): "Integration of social aspects in decision support, based in life cycle thinking". Sustainability, 3, 562-577. DOI:10.3390/su3040562.

Lizcano, E. (1996): "La construcción de la retórica de la imagen pública de la tecnociencia: impactos, invasiones y otras metáforas”. Política y Sociedad, 23: 137-146.

Disponible

en http://revistas.ucm.es/index.php/POSO/article/view/POSO9696330137A/ 25326 (Consulta: 22 mayo 2013)

López Cerezo, J.A. (1998): "Ciencia, tecnología y sociedad ante la educación: El estado de la cuestión en Europa y Estado Unidos”. Revista iberoamericana de educación, $\quad 18$ : 41-68. $\quad$ Disponible http://www.rieoei.org/oeivirt/rie18a02.pdf

López Cerezo, J.A. y Lujan, J.L (2002): “Observaciones sobre indicadores de impacto social”. Revista iberoamericana de ciencia, tecnología, sociedad e innovación. 3. Disponible en http://www.oei.es/revistactsi/numero3/art03.htm (Consulta: 22 mayo 2013)

López Cerezo,J.A.; Méndez Sanz, J.A. y Todt, O. (1998): "Participación pública en política tecnológica: problemas y perspectivas”. Arbor, 627: 279-308. Disponible en http://www.oei.es/salactsi/arbor.htm (Consulta: 22 mayo 2013)

López Cerezo, J.A. y González García, M.I. (2002): Políticas del bosque. Expertos, políticos y ciudadanos en la polémica del eucalipto en Asturias. Cambridge University Press. Madrid.

Luján, J.L. y López Cerezo, J.A. (2003): “La dimensión social de la tecnología y el principio de precaución”. Política y sociedad, 40, 3: 53-60. Disponible en: http://revistas.ucm.es/index.php/POSO/article/view/POSO0303330053A/ 23449 (Consulta: 22 mayo 2013)

Manhart, A., y Griesshammer, R. (2006): Social impacts of the production of notebook PCs, Freiburg, Öko-Institut. Disponible en 
http://www.prosa.org/fileadmin/user_upload/pdf/notebooksurvey_final_e ngl.pdf (Consulta: 22 mayo 2013)

Manuilova, A.; Suebsiri, J. y Wilson, M. (2009): "Should life cycle assessment be part of the environmental impact assessment? Case study: EIA of $\mathrm{CO}_{2}$ capture and storage in Canada”. Energy procedia, 1: 4511-4518. DOI: 10.1016/j.egypro.2009.02.269.

Ministerio de Asuntos Exteriores (1998): Metodología de Evaluación de la Cooperación Española, Madrid, Secretaría de Estado para la Cooperación Internacional y para Iberoamérica, Ministerios de Asuntos Exteriores. Disponible en: http://www.exteriores.gob.es/Portal/es/SalaDePrensa/Multimedia/Publica cio-

nes/Documents/Cooperacion/Evaluacion/Metodologias/Metodologiade\% 20evaluaci\%C3\%B3n\%20\%20de\%20la\%20Cooperaci\%C3\%B3n\%20Es pa\%C3\%B1ola\%20I\%20completo.pdf (Consulta: 22 mayo 2013)

Ministerio de Asuntos Exteriores (2001): Metodología de Evaluación de la Cooperación Española II, Madrid, Secretaría de Estado para la Cooperación Internacional y para Iberoamérica, Ministerios de Asuntos Exteriores. Disponible en: http://www.aecid.es/Centro-

Documentacion/Documentos/Evaluaci\%C3\%B3n/Metodologia2.pdf (Consulta: 22 mayo 2013)

Ministerio de Asuntos Exteriores y Cooperación (2007): Manual de gestión de evaluaciones de la cooperación española: aprender para mejorar, Madrid, Dirección General de Planificación y Evaluación de Políticas para el Desarrollo, Ministerio de Asuntos Exteriores y Cooperación. Disponible en: http://www.exteriores.gob.es/Portal/es/SalaDePrensa/Multimedia/Publicaciones/ Documents/Cooperacion/Evaluacion/Metodologias/Manualdegesti\%C3\%B3ndeevalu aciones.pdf (Consulta: 22 mayo 2013)

Ministerio de Asuntos Exteriores y de Cooperación (2010): La Evaluación en la Cooperación Española. Informe Anual 2009, Madrid, Dirección General de Planificación y Evaluación de Políticas para el Desarrollo, Ministerio de Asuntos Exteriores y Cooperación. Disponible http://www.exteriores.gob.es/Portal/es/SalaDePrensa/Multimedia/Publica ciones/Documents/Cooperacion/Evaluacion/EvaluacionCooperacionES/Infor me\%20Evaluacion\%20en\%20CE_2009.pdf (Consulta: 22 mayo 2013)

Moñux, D., Aleixandre, G., Gómez, F.J. y Miguel, L.J. (2003): Evaluación del impacto social de proyectos de I+D+I: guía práctica para centros tecnológicos, Valladolid, CARTIF y Universidad de Valladolid. Disponible en: http://www.emp.uva.es/ javier/pagina/pantallas/investigacion/evaluacion. pdf

Muñoz-Alonso López, G. (1997): "La evaluación de tecnologías (ET): origen y desarrollo". Revista General de Información y Documentación, 7, 1: 15-30. Disponible 
http://revistas.ucm.es/index.php/RGID/article/view/RGID9797120015A (Consulta: 22 mayo 2013)

NOKIA (2012): NOKIA People \& Planet Report 2012, Nokia, Helsinki. Disponible en http://i.nokia.com/blob/view/-/2438384/data/1/-/NOKIA-PEOPLEPLANET--REPORT-2012-pdf.pdf (Consulta: 22 mayo 2013)

Oakley, P., Pratt, B., y Clayton, A. (1998): Outcomes and Impact: Evaluating Change in Social Development. NGO Management and Policy Series, Oxford, INTRAC.

OECD (2010): Science, Technology and Industry Outlook 2010, Paris, OECD.

OECD DAC Network (2010): Evaluating Development Co-operation. Summary of key norms and standards. Paris. OECD. Disponible en: http://www.oecd.org/dataoecd/12/56/41612905.pdf (Consulta: 22 mayo 2013)

Osuna, J.L., Grávalos, E. y Palacios, C. (2003): Modelos de protocolos para la evaluación de actividades de I+D e innovación, Madrid, FECYT. Disponible en: http://icono.fecyt.es/informesypublicaciones/Documents/modelo_protocol os.pdf (Consulta: 22 mayo 2013)

Pardo Buendía, M. (2002): La evaluación del impacto ambiental y social para el siglo XXI. Teorías, procesos, metodología, Madrid, Editorial Fundamentos.

Pedrosa, R.; Miranda, B.; Aleixandre, G.; Moñux, D. y Gómez, F.J. (2007): “La evaluación del impacto social de las políticas regionales de I+D+i: hacia una lista de control." Estudios de Economía Aplicada. 25, 1: 215-244. Dispone en: http://www.revista-eea.net/documentos/25106.pdf (Consulta: 22 mayo 2013)

Porter, A.L. (1995): "Technology assessment". Impact assessment, 13: 135-151. Disponible en: http://www.hardystevenson.com/Articles/Technology Assessment.pdf (Consulta: 22 mayo 2013)

PROSUITE PROYECT (2013): Handbook on a novel methodology for the sustainability impact assessment of new technologies. SYMLOG. Utrecht. Disponible en: http://www.prosuite.org/c/document_library/get_file?uuid=15c13692d3d4-46ab-9eb2-3d7ce5e9ca63\&groupId=12772 (Consulta: 22 mayo 2013)

Quintanilla, M.A. (1998): “Técnica y cultura”. Teorema, XVII, 3: 49-69. Disponible en: http://sammelpunkt.philo.at:8080/1275/1/QUINTANILLA.pdf (Consulta: 22 mayo 2013)

Riddell, R. C., Kruse, S.E., Kyllonen, T., Ojanper, S. y Vielajus, J.L. (1997): Searching for Impact and Methods: NGO Evaluation, Synthesis Study (Synthesis report), Helsinki, Ministry for Foreign Affairs of Finland. Disponible en http://pdf.usaid.gov/pdf_docs/PNACD416.pdf (Consulta: 22 mayo 2013)

Rip, A. (2003): “Societal Challenges for R\&D Evaluation”, En Shapira, P. y Kuhlmann, S. (eds.) (2003), Learning from Science and Technology Policy Evaluation. Experiences from the United States and Europe, Edward Elgar, Cheltenham-Northampton: 32-53.

Roche, C. (2004): Evaluación del impacto para agencias de desarrollo. Cuadernos de Cooperación, Barcelona, Intermón Oxfam. 
Rodríguez-Carmona Velasco, A. (2002): Las ONG como agentes de desarrollo: la cadena de la ayuda y los procesos de aprendizaje y formación de capital social: un estudio de caso: la experiencia del proyecto PN-23 de Care Bolivia en Villa Serrano, Tesis doctoral, Madrid, Universidad Complutense de Madrid. Disponible en: http://pendientedemigracion.ucm.es/BUCM/tesis/cee/ucm-t26117.pdf (Consulta: 22 mayo de 2013).

Sacristan Navarro, S y Gómez Anson, S. (2009): “CSR in Spain: examples of some practices”. En Mallin, C.A.: Corporate Social Responsibility. A Case Study Approach. Cheltenham, Edward Elgar. 40-58.

Schmidt, I., Meurer, M., Saling, P., Kicherer, A., Reuter, W. y Gensch, C.O. (2004): "SEEBALANCE. Managing Sustainability of Products and Processes with the Socio-Eco-Efficiency Analysis by BASF”. Greener Management International, 45: 79-94.

STOA Science and Technology Options Assessment (2014) Potential and Impacts of Cloud Computing Services and Social Network Sites, Options Brief No 201401. Disponible en http://www.europarl.europa.eu/stoa/webdav/site/cms/shared/4_publicatio ns/Options\%20Brief/STOA\%20Cloud\%20computing\%200ptions\%20bri ef.pdf (Consulta: 22 mayo 2013)

Tran, T.A. (2007): Review of Methods and Tools Applied in Technology Assessment Literature. PICMET 2007 Proceedings, 5-9 August, Portland, Oregon. PICMET. Portland.

Tukker, A. (2000): "Life cycle assessment as a tool in environmental impact assessment". Environmental impact assessment review, 20: 435-456. DOI: 10.1016/j.bbr.2011.03.031

Vanclay, F. (2003): “International Principles for Social Impact Assessment”. Impact Assessment and Project Appraisal, 21, 1: 5-12. DOI: $10.3152 / 147154603781766491$ 